This item was submitted to Loughborough's Research Repository by the author.

Items in Figshare are protected by copyright, with all rights reserved, unless otherwise indicated.

\title{
Minimising peak forces at the shoulders during backward longswings on rings
}

PLEASE CITE THE PUBLISHED VERSION

PUBLISHER

(C) Elsevier

VERSION

AM (Accepted Manuscript)

LICENCE

CC BY-NC-ND 4.0

\section{REPOSITORY RECORD}

Brewin, Mark A., Maurice R. Yeadon, and David G. Kerwin. 2019. "Minimising Peak Forces at the Shoulders During Backward Longswings on Rings". figshare. https://hdl.handle.net/2134/6011. 
This item was submitted to Loughborough's Institutional Repository (https://dspace.lboro.ac.uk/) by the author and is made available under the following Creative Commons Licence conditions.

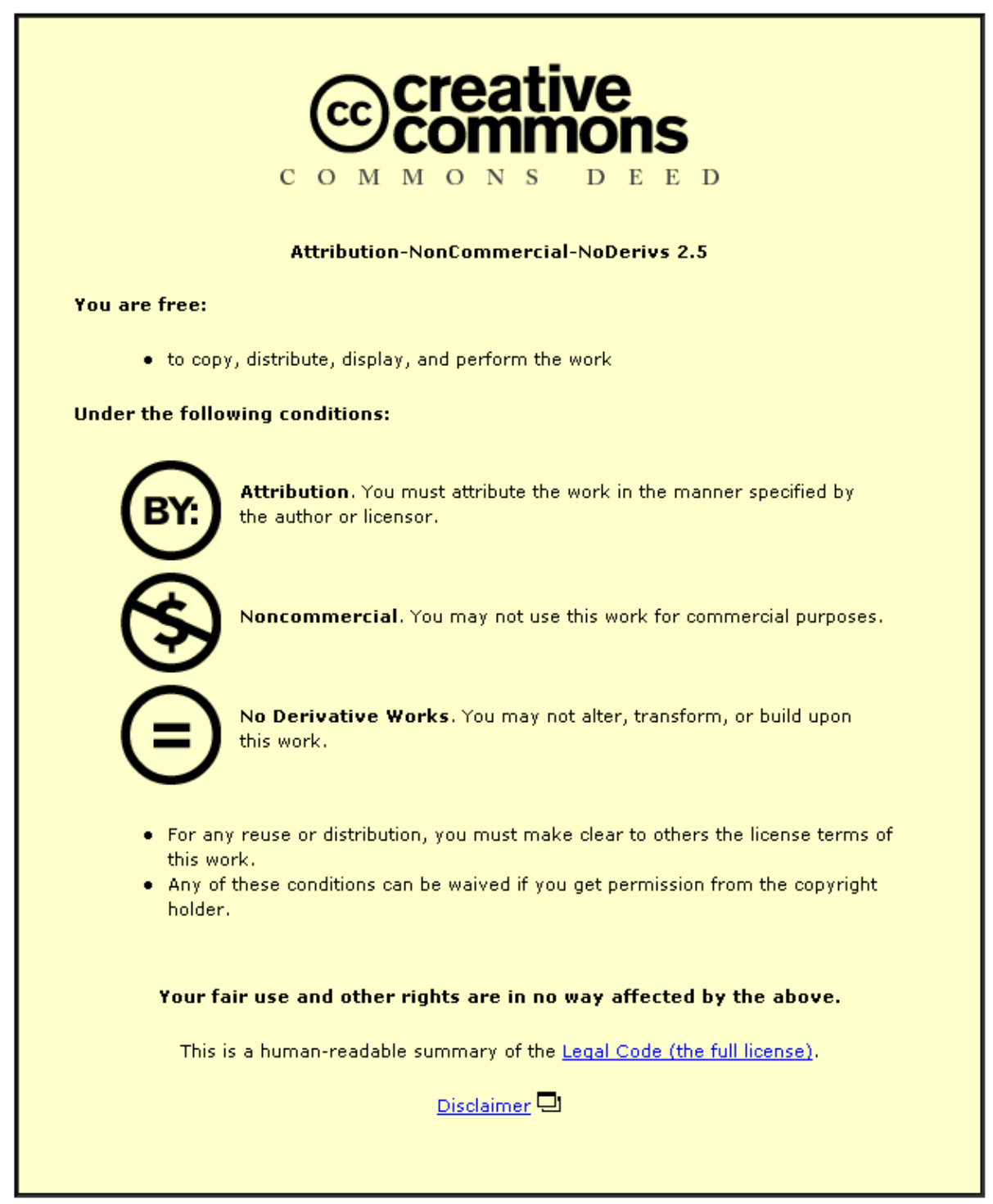

For the full text of this licence, please go to: http://creativecommons.org/licenses/by-nc-nd/2.5/ 


\title{
Minimising peak forces at the shoulders during backward longswings on rings
}

\author{
Mark A. Brewin ${ }^{1}$, Maurice R. Yeadon ${ }^{1}$ and David G. Kerwin ${ }^{2}$ \\ ${ }^{1}$ Department of Sports Science, Loughborough University, Loughborough, UK \\ ${ }^{2}$ Department of Sport and Exercise Science, University of Bath, Bath, UK
}

Abstract

Many elite gymnasts perform the straight arm backward longswing on rings during their competition routines in order to satisfy specific judging requirements. Measured peak combined cable tension during a backward longswing is typically in excess of 9 bodyweights (Nissinen, 1983) and forces of this magnitude have been associated with an increased risk of injury to gymnasts' shoulders (Carraffa et al., 1996). This study investigated the contribution of longswing technique and the elasticity of the gymnast and rings apparatus to minimising loading at the shoulders. A three-dimensional video and cable tension analysis was conducted on a backward longswing performed by an elite gymnast. This analysis provided information regarding the motion of the rings cables, the gymnast's technique and the elasticity of the rings apparatus. A three-dimensional five segment computer simulation model of a gymnast swinging on rings was developed. The inertial characteristics for the model were determined from anthropometric measurements of the gymnast and measurements taken directly from the rings apparatus. The simulation model was evaluated by comparing the backward longswing from the data collection with a simulation of the same performance. The root mean squared differences between the actual performance of the longswing and the evaluated simulation for cable tension and orientations of the gymnast and rings cables were $6.2 \%, 1.0 \%$ and $1.9 \%$ respectively. During the evaluated longswing the peak combined force at the shoulders was 8.5 bodyweights. Modifications to the evaluated simulation of the longswing were used to determine the effect of the gymnast's technique, his elasticity and that of the rings apparatus on peak net shoulder forces. Altering the gymnast's technique, by fixing the gymnast in a straight body configuration throughout the swing, increased the peak shoulder force by 2.56 bodyweights. Removing lateral arm movements, which form part of the gymnast's technique, also resulted in an increased peak shoulder force ( 0.73 bodyweights). Removing the elasticity of the apparatus and gymnast in turn resulted in smaller increases in peak shoulder force ( 0.62 and 0.53 bodyweights). When both aspects of technique were altered the increase in peak shoulder force was 2.5 times greater than when both components of elasticity were removed.

Although the elasticity of the gymnast and apparatus contribute to minimising peak shoulder forces, this study shows that the contribution of a gymnast's technique is considerably greater.

\section{Introduction}

The rings apparatus forms one of six disciplines in the sport of Men's Artistic Gymnastics. Judging criteria stipulate that elite gymnasts must perform a combination of strength and swinging elements throughout their routines on rings. Additional criteria specify that gymnasts must execute both backward and forward swinging elements that are completed in stationary handstand positions (F.I.G., 1997). Since the straight arm backward longswing (Fig. 1, a-i) fulfils these criteria this swinging element is performed by many elite gymnasts in competition. Essentially the backward longswing comprises five phases: an initial handstand with the rings cables in a near vertical orientation (a), a descending phase during which a gymnast adopts an arched body shape as the rings cables initially rotate in the same direction as the longswing (b-d), a swing beneath the rings (e), an ascending phase during which the gymnast adopts a piked configuration as the rings cables initially rotate in the opposite direction to the longswing (f-h), and the final handstand where the rings cables return to a near vertical orientation (i). 


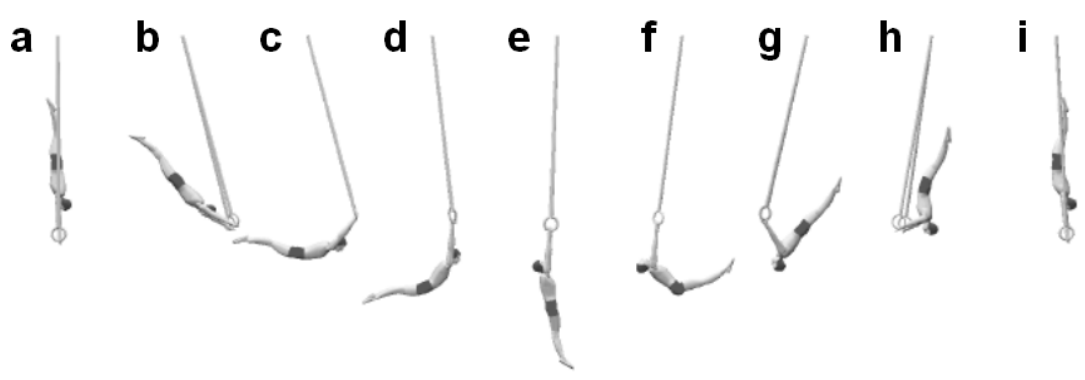

Fig. 1. Graphics sequence of an elite gymnast performing a backward longswing on rings.

In contrast to backward giant circles performed on horizontal bar, where the motions of the gymnast are predominantly parallel to the vertical somersault plane (Brüggemann, 1994), during a backward longswing on rings a gymnast typically moves his arms laterally during the descending and ascending phases of the skill (Fig. 1, c, g). Although such an observation suggests that three-dimensional analyses of this swinging element are necessary, previous experimental and theoretical studies have used only planar analyses (Nissinen, 1983; Brüggemann, 1987; Sprigings et al., 1998). Consequently, previous research may have neglected an important aspect of the backward longswing and may provide only a partial understanding of the factors contributing to the performance of this movement.

During performances of backward longswings by elite gymnasts the peak combined tension measured in the rings cables is typically close to 9 bodyweights (Nissinen, 1983) and occurs as the gymnast passes through the bottom of the swing (Fig. 1, e) in an extended body configuration (Brüggemann, 1987). The large peak cable tension results in the gymnast experiencing large net internal forces at the shoulder joints during this phase of the longswing. Using two-dimensional inverse dynamics analysis Brüggemann (1987) estimated the peak net internal force at each shoulder to be greater than $2100 \mathrm{~N}$.

Several researchers have suggested that peak internal loading of this magnitude in the shoulders increases the risk of ligament and muscular damage and is a cause of increased pain in this region (Brüggemann, 1987; Nissinen, 1995; Caraffa et al., 1996). Such speculations have led to the governing body of gymnastics, the Fédération Internationale de Gymnastique (F.I.G.), providing guidelines to manufacturers of gymnastics apparatus stating that the rings apparatus must possess elastic properties in an attempt to protect the gymnasts' joints and decrease the potential for injury (F.I.G., 1989). At present elastic properties are introduced into many competition rings apparatus via damped elastic devices (DEDs) placed in series within the rings cables and located on top of the horizontal beam of the rings frame. The F.I.G. guidelines and responses from manufacturers highlight the intuitive belief that apparatus elasticity is an important factor in decreasing the net peak internal loading of gymnasts' joints. Indeed, Caraffa et al. (1996) proposed that by further increasing the elasticity of the rings apparatus from present competition norms, the peak net internal loading at the shoulders would be decreased, resulting in a decreased injury risk. However, at present little data exists indicating the relative importance of apparatus elasticity in decreasing peak net internal forces at the shoulders, or to what extent additional elasticity would result in a decreased peak loading.

Using a planar computer simulation model of a gymnast swinging on a rigid rings apparatus Sprigings et al. (1998) provided evidence indicating the potential importance of a gymnast's technique in decreasing the net internal loading at the shoulders during longswings. When the model remained in an extended configuration prior to and throughout the bottom of the swing, peak cable tension exceeded 30 bodyweights. When a hypothetical arched configuration was adopted during the descending phase of the swing, the body became straight and then piked when swinging beneath the rings and the peak combined cable tension was reduced to 12 bodyweights. However, since the model was evaluated only in a qualitative manner, was planar and inelastic in structure, 
and utilised only hypothetical techniques, an accurate assessment of the role of technique in minimising peak shoulder forces cannot be made.

Currently, the literature indicates that at least two methods exist for altering peak net internal forces experienced by gymnasts at the shoulders during longswings on rings: elasticity and technique. However, without a thorough understanding of such factors, unsubstantiated assumptions may be made about their relative contributions to peak shoulder forces and inappropriate suggestions may be offered regarding their potential for further decreasing peak loading. The purpose of this study was to investigate the contributions of apparatus elasticity, gymnast elasticity and technique of a gymnast to minimising peak forces at the shoulders during backward longswings on rings.

\section{Methods}

In order to obtain three-dimensional kinetic and kinematic data for a performance of a backward longswing on rings a force and video data collection session was conducted. Information gained from this analysis was subsequently used to define the structure of a three-dimensional simulation model, to determine estimates of simulation model parameters and to evaluate the model, as described in this section.

\section{Data Collection}

A synchronised three-dimensional video and cable tension analysis was conducted on a backward longswing performed by an elite male gymnast in the training environment. The attire of the subject comprised a leotard and contrasting shorts only and informed consent was given by the subject prior to the data collection. Anthropometric data were collected on the subject using the procedure outlined by Yeadon (1990b) for the determination of personalised segmental inertia parameters.

A specially constructed rings cable, with a calibrated Kistler quartz force link (9331A) placed in series within the cable, was positioned in a standard competition rings apparatus to measure cable tension throughout a longswing. Since Nissinen (1983) reported similar magnitudes of tension in both rings cables during performances of longswings, the measured tension was doubled to provide combined cable tension. The analogue signal from the force link was transmitted to a Kistler charge amplifier (C5007) and subsequently passed into an unsigned 12 bit analogue to digital converter (CED1401) sampling at $1000 \mathrm{~Hz}$. The digital output was recorded onto the hard disc of an A5000 Acorn computer and converted into ASCII format. The collection of force data was initiated by a two-way manual trigger and lasted 5 seconds, with 1.5 seconds of peri-triggering. The trigger was pressed as the gymnast started the descending phase of the longswing. The two-way trigger also simultaneously illuminated an array of 18 light emitting diodes (LED), which was used to synchronise force and video data to within half a video field.

The longswings were video-recorded using two genlocked Hi 8 video cameras, a Sony HyperHAD (EVW-300P) and a Sony HandyPRO (CCD-VX1E), recording at $50 \mathrm{~Hz}$ with electronic shutter speeds of $1 / 250 \mathrm{~s}$ and $1 / 300 \mathrm{~s}$ respectively. The cameras were positioned on balconies $4.5 \mathrm{~m}$ above the floor level and at distances of $36 \mathrm{~m}$ and $25 \mathrm{~m}$ from the rings frame respectively. The horizontal field of view for each camera was approximately $8 \mathrm{~m}$. Fig. 2 shows a plan view of the locations of the cameras relative to the rings frame, together with the location of the synchronisation LED array. 


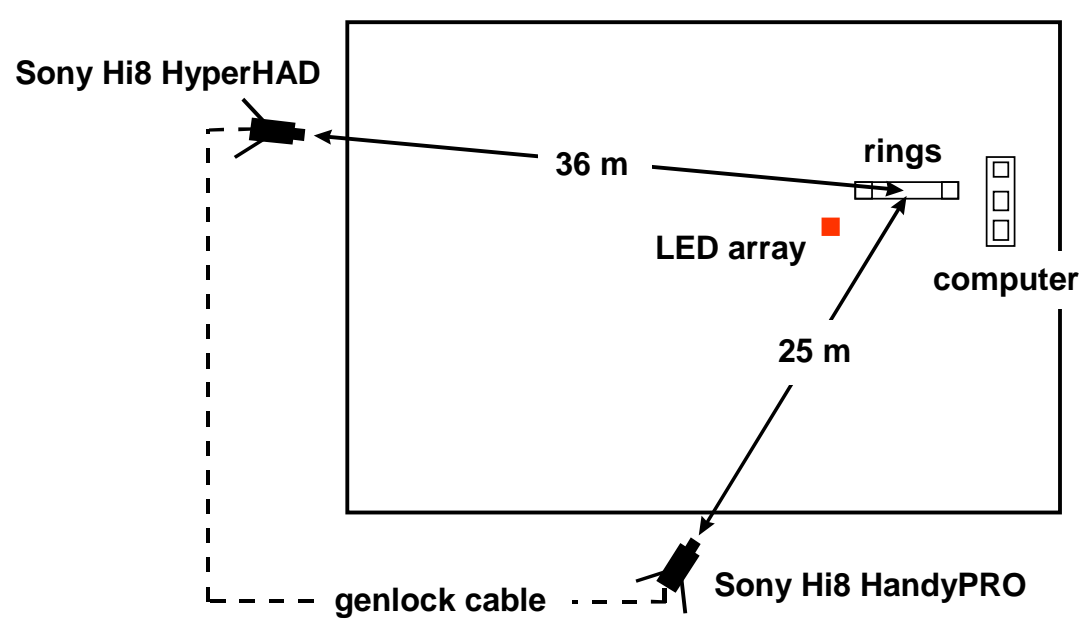

Fig. 2. A plan view diagram indicating the camera locations relative to the rings apparatus for the force and video data collection.

Prior to the backward longswing performances a calibration structure was centred between the uprights of the rings frame and was video-recorded simultaneously from both camera views. The structure comprised 12 calibration points of known location and spanned a volume measuring $5.7 \mathrm{~m}$ high by $1.8 \mathrm{~m}$ wide by $1.8 \mathrm{~m}$ deep. The calibration volume included the majority of the motion of the gymnast and apparatus.

After removing the calibration structure, the subject performed several backward longswings from a near motionless initial handstand. The proficiency of each longswing was assessed by a national coach and acceptable performances were noted. The gymnast was allowed to rest between trials in order to minimise the effect of fatigue. A longswing deemed proficient by the national coach was used in the following analysis.

\section{Data Processing}

The anthropometric data for the subject was used with the mathematical inertia model of Yeadon (1990b) to determine personalised segmental inertia parameters for both the video analysis and the simulation model. To attenuate systematic and random noise in the cable tension data, frequencies greater than $45 \mathrm{~Hz}$ were removed from the raw cable tension time history using a truncated Fourier series.

The video images were converted from Hi8 to sVHS and digitised using the TARGET high resolution video digitising system (Kerwin, 1995). The 12 calibration markers were digitised from each camera view over five consecutive fields. The mean digitised coordinate for each marker was used to represent the digitised location. For the longswing sequence 15 body landmarks on the gymnast were digitised: the left and right wrist, elbow, shoulder, hip, knee and ankle joint centres, and the centre of the head. Six landmarks on the rings apparatus were also digitised during the longswing: the left and right hands grasping the rings, the pivot attachments of the rings cables and the damped elastic devices on top of the horizontal beam of the rings frame. The first digitised field was approximately 0.5 seconds prior to the gymnast initiating the descent and the final field was approximately 1.0 second after the gymnast first attained the final handstand position.

For each camera view, the digitised data for the calibration markers were combined with their known locations to calibrate the camera-digitiser system using the direct linear transformation (DLT) procedure (Abdel-Aziz \& Karara, 1971). The three-dimensional locations of the digitised landmarks on the gymnast and rings apparatus were subsequently reconstructed using the calibrated camera-digitiser system and the DLT procedure (Abdel-Aziz \& Karara, 1971).

The method described by Yeadon (1990a), which utilises mutually perpendicular axes fixed in segments and successive ordered rotations about these axes, was used to determine body configuration and orientation angles of the gymnast and rings cables and the mass centre location of the gymnast. The following body segments were defined from the digitised articulations of the 
gymnast: shanks with feet, thighs, torso with head, and arms. From these body segments four angles were calculated to describe the configuration of the gymnast. The knee angle defined the angle between the shanks and the thighs and the hip angle described the angle between the thighs and the torso. Two angles, arm elevation and arm abduction, defined the three-dimensional configuration of the arms relative to the torso of the gymnast. The arm abduction angle represented the lateral movements of the gymnast's arms. For each configuration angle the average of the left and right sides values was calculated from the video data and was used to represent the gymnast's configuration throughout the longswing. The wrist to ankle length, calculated by the summation of the shank, thigh, torso and arm segment lengths, provided information about the extension of the gymnast throughout the longswing.

The orientation of the gymnast in the inertial reference frame was defined by the angle of the torso from the vertical. An additional angle, termed the body angle $\varepsilon$, which described the overall orientation of the gymnast was calculated as the angle between the vertical and a line joining the gymnast's mass centre to the midpoint of the hands (Fig. 3). In the initial handstand both the torso angle and body angle were close to $0^{\circ}$. On completion of the backward longswing these angles were close to $360^{\circ}$.

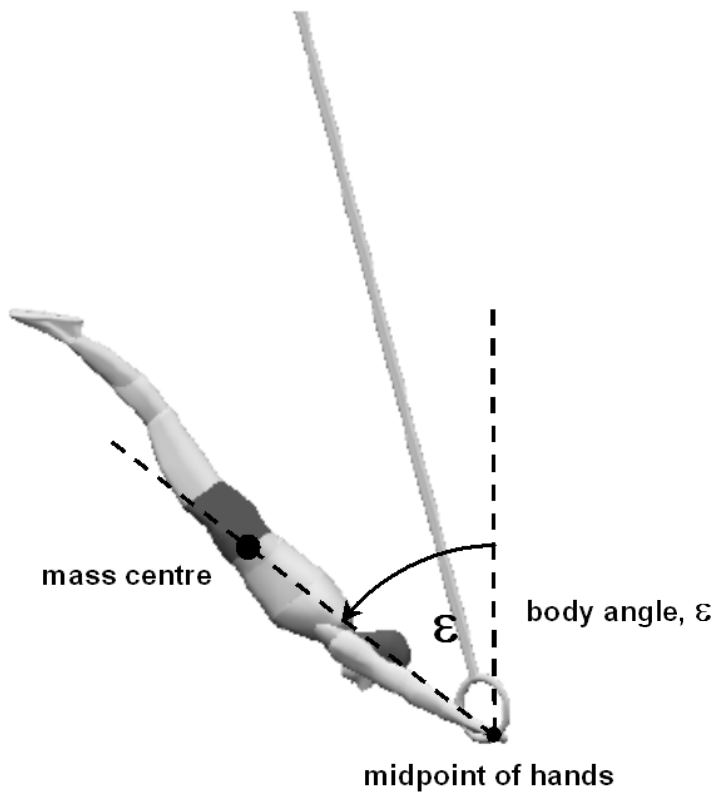

Fig. 3. Definition of the whole body orientation angle, termed the body angle $(\varepsilon)$.

The rings cables were defined by a vector from the gymnast's hands to the pivot attachments of the cables. The cable orientations were defined in the inertial reference frame by two successive ordered angles: cable elevation and cable abduction. The elevation angle described the forward and backward motion of the cables while the abduction angle represented the lateral movement. The average angles for the left and right rings cables were used to represent their motion. The length of the rings cables and the horizontal and vertical movements of the DEDs were also calculated throughout the longswing.

The configuration and orientation angles for the gymnast and rings cables, together with the extension of the gymnast and the deformations of the rings apparatus, were fitted using Jennings' quintic spline routine (Wood \& Jennings, 1979) to obtain continuous time histories and first and second derivatives.

\section{Data Analysis}

The stiffness and damping coefficients of the DEDs were estimated using linear regression of the vertical component of cable tension as a function of the vertical displacement and velocity of the 
DEDs. A similar multiple linear regression was performed for the rings cables to estimate their stiffness and damping. The stiffness and damping coefficients associated with the horizontal motion of the rings frame were estimated by assuming that an effective mass of the rings frame (calculated to be $33.5 \mathrm{~kg}$ ) was undergoing damped simple harmonic motion. These analyses provided initial estimates of the elasticity of the rings apparatus for the simulation model.

\section{The Simulation Model}

AUTOLEV ${ }^{\mathrm{TM}} 3$, a software package for dynamics, was used to produce the three-dimensional simulation model of a gymnast swinging on rings. AUTOLEV ${ }^{\mathrm{TM}} 3$ utilises Kane's method for formulating the equations of motion for a mutli-link system. For a comprehensive review of Kane's method the reader is directed to Kane, Linkins \& Levinson (1983).

The model comprised segments representing both the rings apparatus and the gymnast (Fig. 4). Constraint equations were used to ensure the motion of the model was symmetrical about the sagittal plane. The rings frame was represented by a large effective mass $(33.5 \mathrm{~kg})$ at the top of the rings cables, while the cables were represented by two rigid segments. The cables were able to rotate in three dimensions, described by two orientation angles: cable elevation and abduction. The inertial characteristics of the rings cables and rings frame were based on the actual rings apparatus used in the data collection. Damped linear springs represented the elasticity of the rings frame in the horizontal direction, the DEDs in the vertical direction and the elasticity of the rings cables.

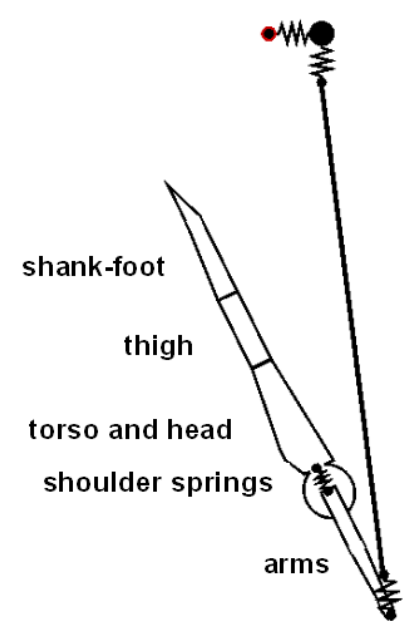

lateral view

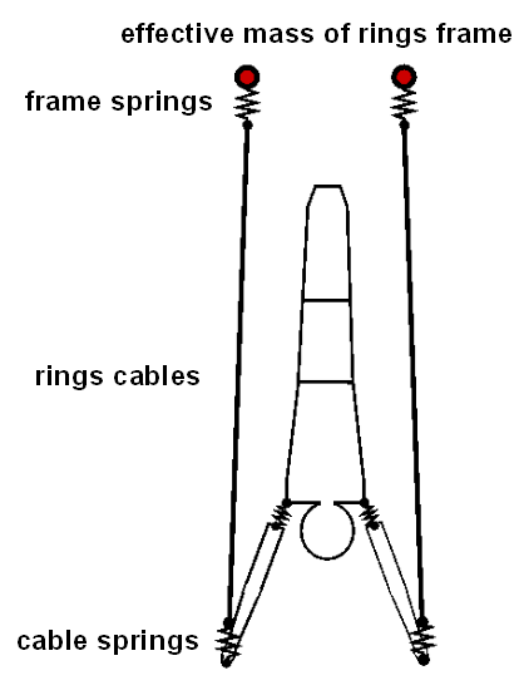

frontal view

Fig. 4. Lateral and frontal views indicating the structure of the three-dimensional simulation model of a gymnast swinging on rings.

The modelled gymnast comprised five rigid segments: two arms, a torso with head, thigh and shank-foot (Fig. 4). The inertial characteristics of the modelled gymnast were based on the values calculated for the subject in the data collection using the mathematical model of Yeadon (1990b). The orientation of the model was defined by the torso angle while the configuration was defined by four joint angles which gave the relative orientations of the thigh and torso, the shank and thigh, and the arms and torso. The body angle $\varepsilon$ of the model was calculated in the same manner as in the video analysis. Non-linear damped springs, with force as a quadratic function of extension, were introduced at the shoulder joints to represent the elastic properties of a gymnast. A Runge-Kutta numerical integration algorithm with variable step length was used to advance the solution of the equations of motion for the model.

Input to the simulation model included the technique throughout a simulation, described in terms of the time histories of the four body configuration angles and their first two derivatives. Initial conditions required by the model included the angle and angular velocities of the model's torso and rings cables and the lengths and velocities of all the springs. Output from the model 
included the resulting motion of the rings cables and gymnast, the change in wrist to ankle length of the gymnast, the combined cable tension and the peak forces at the shoulder joints.

\section{Evaluation of the Simulation Model}

In order to assess the accuracy of the simulation model it was evaluated against the backward longswing analysed in the data collection. The technique of the gymnast from the data collection, as described by the four configuration angles throughout the longswing, was used to drive the simulation model. Initial angles and angular velocities of the model's torso $\left(5.8^{\circ}\right.$ and $\left.0.49 \mathrm{rad}^{-1} \mathrm{~s}^{-1}\right)$ and elevation of the rings cables $\left(1.7^{\circ}\right.$ and $\left.0.21 \mathrm{rad}_{\mathrm{s}} \mathrm{s}^{-1}\right)$ were taken from the actual performance. The initial lengths of the apparatus springs were based on the measured cable tension data, while the initial lengths of the shoulder springs were based on the gymnast being in a handstand. Since the gymnast was initially close to a stationary handstand position the initial velocities of all springs were set to $0 \mathrm{~m} . \mathrm{s}^{-1}$. The evaluation was performed from an initial body angle $\varepsilon$ of $5^{\circ}$ (angular velocity $0.5 \mathrm{rad} . \mathrm{s}^{-1}$ ) to a final body angle of $360^{\circ}$, and therefore incorporated the entire swinging phase of the backward longswing.

The analysis of cable tension and deformations of the rings apparatus from the data collection provided initial estimates for the stiffness and damping of the rings apparatus. However, no estimates were obtained for the stiffness and damping of the gymnast's shoulders. In order to obtain more accurate spring parameter estimates for the apparatus and obtain spring parameters for the gymnast's shoulders, an optimisation procedure was employed. A function describing the overall difference between the actual and simulated performance was calculated as the rms difference between body angle, cable elevation angle, cable tension and wrist to ankle length throughout the longswing. Weightings for each component, based on the magnitude of their total excursions during the actual performance, were calculated in order to provide equal emphasis in the function. The spring parameters (stiffness and damping for the horizontal frame, DEDs, cables and shoulders) were varied within realistic upper and lower boundaries using the simulated annealing optimisation algorithm (Goffe et al., 1994) until the function describing the difference between the actual and simulated performances was minimised.

\section{Simulations investigating contributions to peak shoulder forces}

Seven simulations were conducted using modifications to the evaluated backward longswing to determine the contributions of the gymnast's technique, his elasticity and that of the apparatus to peak shoulder forces. The effect of the modifications on peak shoulder force was determined by the change in peak force between the evaluated and modified simulations.

In three simulations the contribution of the gymnast's technique to peak shoulder forces was determined. In the first simulation the model's hip and shoulder elevation angles were modified to give a fixed extended configuration throughout the swing. In the second simulation all lateral arm movements made by the model were removed. In the third a fixed near straight configuration was maintained throughout the swing and all lateral arm movements were omitted.

Further simulations were used to determine the contribution of the elastic properties of the gymnast and rings apparatus to peak shoulder forces. In a fourth simulation the apparatus (horizontal and vertical frame springs and cable springs) was modelled as a rigid structure. In the fifth simulation the modelled gymnast possessed no elastic properties, while in the sixth simulation all of the elastic elements were modelled as rigid structures.

A final simulation was conducted in which the rings apparatus and modelled gymnast were rigid and the technique comprised a fixed extended configuration without lateral arm movements.

\section{Results}

The following section comprises results from the evaluation of the three-dimensional simulation model and the modified simulations used to investigate the various contributions to minimising peak forces at the shoulders. 


\section{Inertia Parameters for the Model}

Table 1 provides values for the inertial parameters for the apparatus and personalised segmental values for the gymnast in the simulation model.

Table 1. Inertia parameters of the rings cables and body segments in the model

\begin{tabular}{|l|c|c|c|c|c|c|}
\cline { 2 - 7 } \multicolumn{1}{c|}{} & $\begin{array}{c}\text { length } \\
(\mathrm{m})\end{array}$ & $\begin{array}{c}\text { mass } \\
(\mathrm{kg})\end{array}$ & $\begin{array}{c}\text { mass centre } \\
\text { location }(\mathrm{m})\end{array}$ & \multicolumn{3}{|c|}{$\begin{array}{r}\text { principal moments of inertia } \\
\left(\mathrm{kg} . \mathrm{m}^{2}\right)\end{array}$} \\
\hline segment & & & & frontal & transverse & longitudinal \\
\hline each rings cable & 2.923 & 1.000 & 1.461 & 0.712 & 0.712 & 0.001 \\
\hline each arm & 0.607 & 3.888 & 0.355 & 0.127 & 0.127 & 0.004 \\
\hline torso + head & 0.475 & 34.399 & 0.127 & & 1.628 & \\
\hline thigh & 0.410 & 16.289 & 0.175 & & 0.235 & \\
\hline shank-foot & 0.615 & 8.839 & 0.235 & & 0.240 & \\
\hline
\end{tabular}

\section{Evaluation}

The results of the evaluation indicate the overall accuracy of the model when simulating the gymnast performing a backward longswing. The stiffness and damping values for the springs in the model determined from the optimisation procedure are provided in Table 2. These final estimates for the stiffness coefficients of the springs representing the apparatus were within $12 \%$ of the original values estimated from deformations of the rings apparatus.

The graphics sequence shown in Fig. 5 highlights the accuracy in simulating the gymnast's body angle and the elevation angle of the rings cables. The root mean squared (rms) difference between actual and simulated values for these two angles, when expressed as a percentage of their total actual excursions (Table 3), were $1.0 \%$ and $1.9 \%$ respectively.

Table 2. Stiffness and damping coefficients of the springs determined from the optimisation procedure

\begin{tabular}{|l|c|c|c|c|}
\cline { 2 - 5 } \multicolumn{1}{c|}{} & \multicolumn{4}{c|}{ model springs } \\
\cline { 2 - 5 } \multicolumn{1}{c|}{} & horizontal & vertical & cable & shoulder \\
\hline stiffness $\left(N . m^{-1}\right)$ & 20134 & 75416 & 25587 & $22864,9788 *$ \\
\hline damping (N.s.m ${ }^{-1}$ ) & 91 & 4061 & 3505 & 1589 \\
\hline
\end{tabular}

Note: *The shoulder spring is non-linear producing a force of $\mathrm{Ax}^{2}+\mathrm{Bx}$ for an extension $\mathrm{x}$. The table lists the values of the constants A and B respectively. 


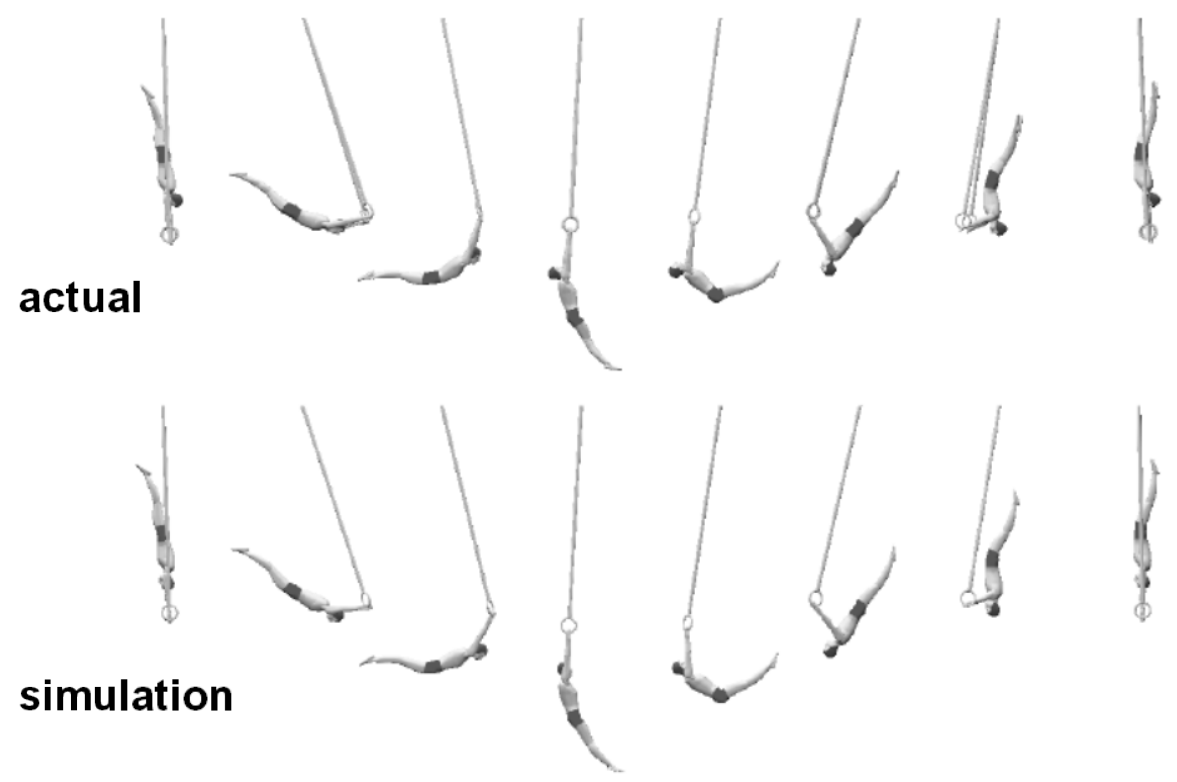

Fig. 5. Graphics sequence comparing the orientations of the gymnast and rings cables for the evaluated backward longswing. The upper sequence is from video derived data, while the lower sequence is the simulated performance.

Table 3. Total excursions of performance variables and root mean square differences between actual and simulated performances

\begin{tabular}{|c|c|c|c|}
\hline \multicolumn{4}{|c|}{ total excursion of variables for actual performance } \\
\hline $\begin{array}{c}\text { body angle } \\
\left(^{\circ}\right)\end{array}$ & $\begin{array}{c}\text { cable elevation angle } \\
\left({ }^{\circ}\right)\end{array}$ & $\begin{array}{c}\text { combined cable tension } \\
(\mathrm{N})\end{array}$ & $\begin{array}{c}\text { wrist-ankle length } \\
(\mathrm{m})\end{array}$ \\
\hline 355.2 & 58.7 & 4813.4 & 0.27 \\
\hline
\end{tabular}

\begin{tabular}{|c|c|c|c|}
\hline \multicolumn{4}{|c|}{ root mean squared difference between actual and simulated performance } \\
\hline $\begin{array}{c}\text { body angle } \\
\left({ }^{\circ}\right)\end{array}$ & $\begin{array}{c}\text { cable elevation angle } \\
\left({ }^{\circ}\right)\end{array}$ & $\begin{array}{c}\text { combined cable tension } \\
(\mathrm{N})\end{array}$ & $\begin{array}{c}\text { wrist-ankle length } \\
(\mathrm{m})\end{array}$ \\
\hline 3.3 & 1.1 & 297.3 & 0.06 \\
\hline
\end{tabular}

Cable tension and wrist to ankle length were also incorporated into the function used in the evaluation procedure. Time histories for the actual and simulated performances for these two variables are shown in graphical form in Fig. 6. The rms difference between measured and simulated cable tension, when expressed as a percentage of the peak measured tension, is $6.2 \%$. The rms difference between measured and simulated values for the wrist to ankle length is $0.059 \mathrm{~m}$ (Table 3) which is equivalent to $22.1 \%$ of the total actual excursion of this variable. 

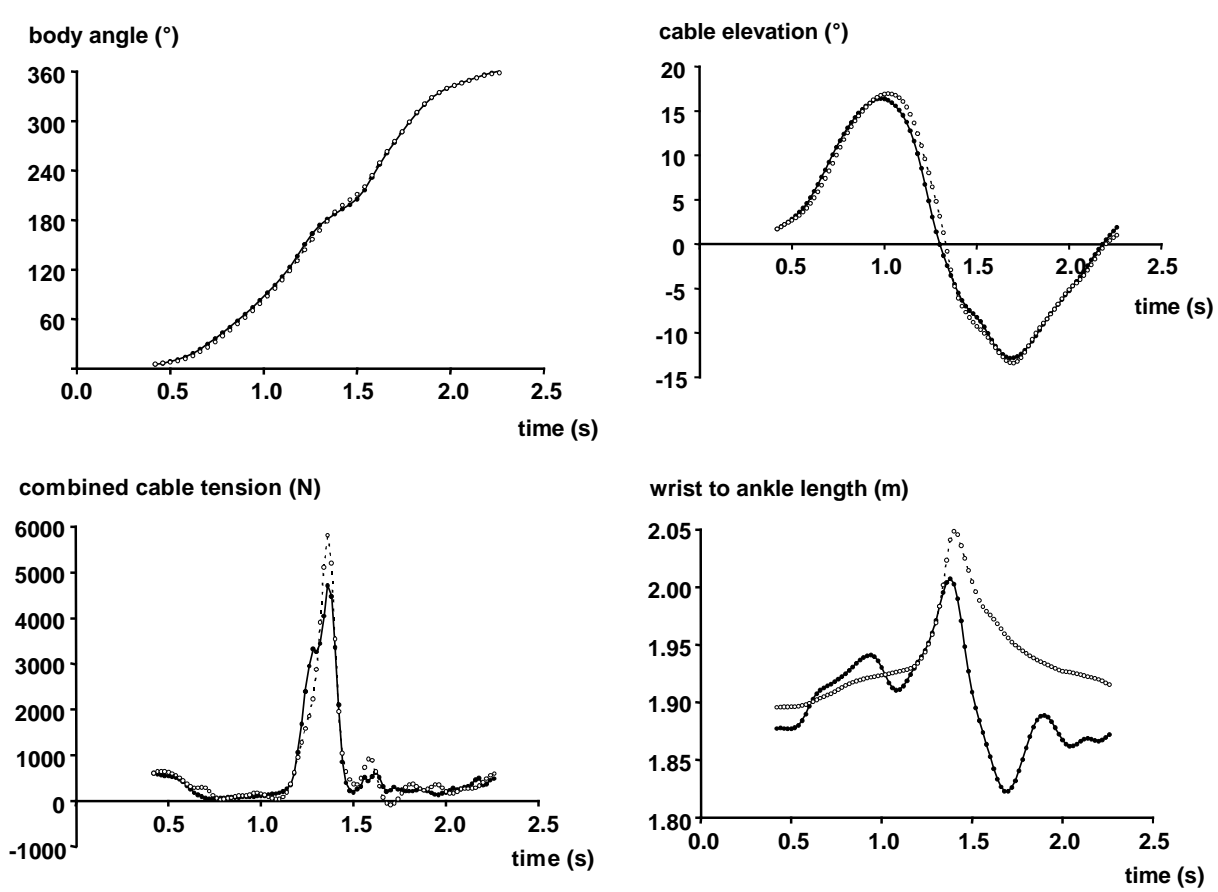

Fig. 6. Time histories of the components included in the optimisation function giving the difference between the actual (closed circles) and simulated (open circles) performance for the evaluated backward longswing (gymnast orientation, cable orientation, combined cable tension and wrist to ankle length).

The peak combined net shoulder force for the evaluated longswing was 8.5 bodyweights, which is equivalent to $2800 \mathrm{~N}$ at each shoulder. The results from the evaluation procedure indicate the three-dimensional simulation model is sufficiently accurate to determine the role of apparatus and gymnast characteristics and the gymnast's technique to minimising peak shoulder forces.

\section{Contributions to minimising peak shoulder forces}

The effects of apparatus elasticity, gymnast elasticity and the gymnast's technique on peak net shoulder forces were determined by implementing modified simulations for the evaluated longswing. Fig. 7 shows the change in peak net shoulder force when aspects of technique (arching and lateral arm movements) and elasticity (apparatus and elasticity) were altered individually and in combination. Altering only the arching-to-piking action of the gymnast's technique, by maintaining the model in an extended configuration, provided the largest increase (2.56 bodyweights) in peak combined shoulder force. The removal of lateral arm movements provided a larger increase in peak shoulder forces ( 0.73 bodyweights) than the removal of elastic properties of either the gymnast or apparatus. The smallest increase in peak force ( 0.53 bodyweights) occurred when only the shoulders were altered to represent a rigid structure. When both aspects of technique were altered the increase in peak shoulder force was 2.5 times greater than when both components of elasticity were removed (Fig. 7). 
a)

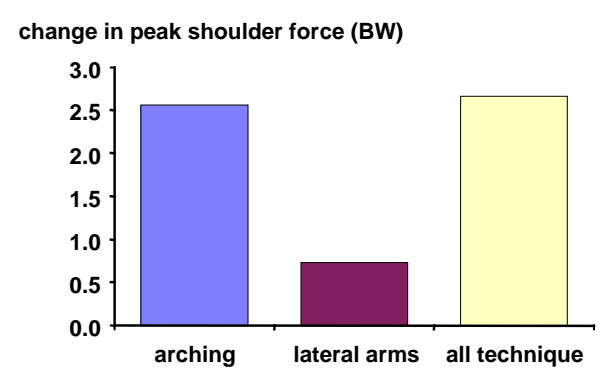

b)

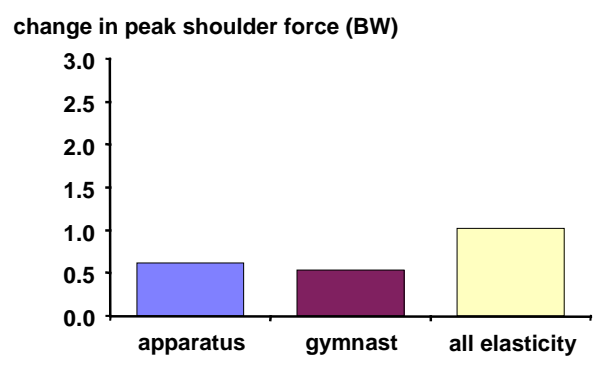

Fig. 7. Changes in combined peak shoulder forces from the evaluated longswing due to (a) changes in the gymnast's technique due to 1) arching, 2) lateral arm movements and 3) arching and lateral arm movements; (b) changes in the elasticity of 1) the gymnast, 2) the apparatus and 3) the gymnast and the apparatus.

Finally, in the hypothetical scenario when the gymnast and apparatus were modelled as rigid structures and both aspects of technique were changed, the peak net combined shoulder force was 24.05 bodyweights, or nearly three times the force in the original simulation. This result indicates the combined importance of the gymnast's technique, his elasticity and the elasticity of the apparatus in minimising peak combined shoulder forces during a backward longswing on rings.

\section{Discussion}

The results of the evaluation indicate that the three-dimensional simulation model gives an accurate representation of a gymnast performing a backward longswing on rings. Unlike the model described by Sprigings et al. (1998), the model developed in the present study includes the lateral arm movements utilised by many elite gymnasts and represents the elastic properties of the both the apparatus and gymnast. By including these features of the actual sporting situation, this study has been able to quantify their contributions to minimising peak shoulder forces to levels which gymnasts are able tolerate without serious injury.

The results from the modified simulations highlight the importance of the gymnast's technique in decreasing net internal forces at the shoulders. Without attaining an arched body shape during the descending phase and rapidly changing through an extended configuration to a piked one early in the ascending phase, the peak shoulder forces would potentially increase by 2.56 bodyweights. The contribution of the arching-to-piking action of the gymnast's technique to decreasing peak forces was identified by Sprigings et al. (1998). The action of changing body configuration from arched through to an extended shape as the loading becomes high increases the duration of high acceleration and reduces the peak vertical acceleration of the mass centre. This results in lower peak tension in the cables and consequently in lower forces experienced by the gymnast at the shoulders. Similarly, although to a lesser extent, if the gymnast removed his lateral arm movements, the peak combined shoulder force would increase by 0.73 bodyweights. This contribution of lateral arm movements, neglected by previous planar studies, may be considered to be important for load minimisation in a backward longswing.

Increases in peak forces of these magnitudes due to the removal of selected aspects of a gymnast's technique are undesirable. If peak internal forces during swinging on rings are a major cause of muscular and ligament injuries in the shoulder region, increases in the magnitude of these forces would be potentially injurious. Thus the arching-to-piking and lateral arm movements adopted by the gymnast in this study contribute to a decreased injury potential. These movements may also assist in the production of rotation required to complete the backward longswing to handstand.

The results from this study were in some agreement with the intuitive belief of the world governing body of gymnastics and the manufacturers of gymnastics equipment that elastic properties in the rings apparatus contribute to a decrease in peak internal forces. However, the reduction of 0.6 bodyweights in peak combined shoulder forces due to the apparatus elasticity is 
relatively small, especially considering the emphasis placed on this factor by manufacturers of gymnastics equipment and the F.I.G..

The importance of the gymnast's elasticity in minimising peak loading at the shoulders ( 0.54 bodyweights) was shown to be similar to that of the apparatus. Although representing the elasticity of the gymnast by non-linear damped springs was found to produce more realistic changes in the wrist to ankle length than simple linear springs, this passive system was unable to simulate the active extension of the gymnast observed during the descending phase of the longswing (Fig. 7, $0.6 \mathrm{~s}-0.9 \mathrm{~s}$ ). To overcome this discrepancy, and potentially increase the accuracy of the model, additional parameters may be incorporated into the model to represent the muscular activation at the shoulders. However, such alterations would further increase the complexity of the model and require more subject specific parameter estimates which are not readily available. Such a development may not be considered beneficial or necessary when considering the minor role of the gymnast's elasticity in minimising peak loading at the shoulders during a longswing.

The final modified simulation, where the gymnast's technique was altered and all elastic components were modelled as rigid structures, may be considered to be unrealistic. However, the result from this simulation highlights the combined importance of technique and elastic properties, since it is most unlikely that a gymnast could tolerate forces as large as 12 bodyweights at each shoulder joint.

In addition to quantifying the contributions to minimising peak shoulder force, the speculation of Caraffa et al. (1996), that peak shoulder forces may be further reduced with an increase in apparatus elasticity from competition norms was also investigated. Using the evaluated simulation model the stiffness of each spring representing the rings apparatus was reduced by one third, providing the apparatus with $50 \%$ more elasticity than the competition apparatus. This relatively large increase in elasticity resulted in a relatively small reduction in net peak shoulder forces of 0.2 bodyweights at each joint. Consequently, the speculation that shoulder injuries may be drastically reduced by increasing the elasticity of the rings apparatus is unfounded.

\section{Conclusion}

The techniques that gymnasts adopt during backward longswings on rings form a major role in minimising the peak shoulder forces they experience. The arching-to-piking aspect of technique provides the largest contribution to limiting peak shoulder forces to a tolerable level, while the role of lateral arm movements may also be considered important. The elastic properties of gymnasts and the rings apparatus do contribute to minimising peak forces, although to a much lesser extent than the technique of a gymnast.

The association in the research literature between peak shoulder forces and injury to this muscular joint means that correct technique and elastic apparatus are essential in reducing the risk of injury to a gymnast. However, from the additional results it may be speculated that it is not possible to further drastically decrease the peak shoulder forces experienced by gymnasts during longswings through modifications to the elasticity of the apparatus only. Through trial and error, elite gymnasts may have already developed appropriate techniques to ensure the peak shoulder forces are usually tolerable for a given apparatus elasticity, while working within the constraints of required technical form to produce a longswing to a final stationary handstand.

\section{Acknowledgements}

The financial support of UK Sport, formerly known as the United Kingdom Sports Council, for this research study is gratefully acknowledged. 


\section{References}

Abdel-Aziz, Y.I., Karara, H.M., 1971. Direct linear transformation from comparator coordinates into object space coordinates in close range photogrammetry. (ASP Symposium on

Close-Range Photogrammetry). Falls Church, VA: American Society of Photogrammetry, pp 3-18.

Brüggemann, G.-P., 1987. Biomechanics in gymnastics. In: Hebbelinck, M., Shephard, R.J (Eds.), Medicine and Sports Science. Basel: Karger, pp 142-176.

Brüggemann, G.-P., 1994. Biomechanics of gymnastics techniques. In: Nelson, R.C., Zatsiorsky, V.M. (Eds.), Sports Science Review: Sport Biomechanics. Champaign, IL: Human Kinetics, pp 79-120.

Caraffa, A., Cerulli, G., Rizzo, A., Buompadre, V., Appoggetti, S., Fortuna, M., $1996 . \quad$ An arthroscopic and electromyographic study of painful shoulders in elite gymnasts. Knee Surgery, Sports Traumatology and Arthroscopy 4, 39-42.

Fédération Internationale de Gymnastique (F.I.G.), 1997. Code of Points. Artistic Gymnastics for Men (1997 edition). Switzerland: F.I.G.

Fédération Internationale de Gymnastique (F.I.G.), 1989. Apparatus norms for official events of artistic gymnastics and rhythmic sportive gymnasts. Switzerland: F.I.G., pp 56-62.

Goffe, W.L., Ferrier, G.D., Rogers, J., 1994. Global optimisation of statistical functions with simulated annealing. Journal of Econometrics 60, 65-99.

Kane, T.R., Likins, P.W., Levinson, D.A., 1983. Spacecraft Dynamics. New York: McGraw-Hill Publishing Company.

Kerwin, D.G., 1995. Apex/Target high resolution video digitising system. In: Watkins J. (Ed.), Proceedings of the Biomechanics Section of the British Association of Sport and Exercise Sciences. Leeds: BASES, pp. 1-4.

Nissinen, M.A., 1983. Kinematic and kinetic analysis of the giant swing on rings. In: Matsui, H., Kobayashi, K,. (Eds.), Biomechanics VIII-B. Champaign, IL: Human Kinetics, pp 781-786.

Nissinen, M.A., 1995. Analysis of reaction forces in gymnastics on the rings. In: Häkkinen, K., Keskinen, K.L., Komi, P.V., Mero, A. (Eds.), Abstracts of the International Society of Biomechanics XV ${ }^{\text {th }}$ Congress. Jyväskylä: ISB, pp 680-681.

Sprigings, E.J., Lanovaz, J.L., Watson, L.G., Russell, K.W., 1998. Removing swing from a handstand on rings using a properly timed backward giant circle: a simulation solution. Journal of Biomechanics 31, 27-35.

Wood, G.A., Jennings, L.S., 1979. On the use of spline functions for data smoothing. Journal of Biomechanics 12, 477-479.

Yeadon, M.R., 1990a. The simulation of aerial movement - I. The determination of orientation angles from film data. Journal of Biomechanics 23, 59-66.

Yeadon, M.R., 1990b. The simulation of aerial movement - II. A mathematical inertia model of the human body. Journal of Biomechanics 23, 67-74. 